\title{
ACTUALITÉS AUC Les urologues en formation : notre avenir
}

\section{Michael P. Leonard, MD, FRCSC}

\section{Président, AUC}

Cite as: Can Urol Assoc J 2015;9(11-12):429 http://dx.doi.org/10.5489/cuai.3482 Published online December 14, 2015.

The CUA exists to promote the highest standard of urologic care for Canadians and to advance the art and science of urology.

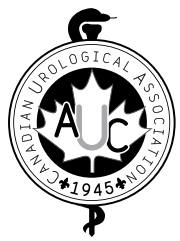

I 'AUC a récemment célébré son $70^{\mathrm{e}}$ anniversaire lors d'un congrès très agréable à Ottawa. Le retour sur le passé a son importance, bien sûr, mais il est essentiel pour notre organisme de garder un œil sur l'avenir de l'urologie au Canada, un avenir qui repose sur les urologues en formation dans les facultés de médecine partout au pays.

L'AUC déploie s'efforce consciemment d'assurer sa pertinence pour ces futurs collègues tout au long des différentes phases de leur éducation et leur passage à l'exercice de la profession. Nos efforts débutent par des vidéos affichées sur YouTube produites par l'AUC et reliées au site web de l'AUC (www.cua.org), vidéos où des urologues canadiens et des urologues en formation tentent d'attirer vers notre spécialité la crème de la crème des étudiants. En outre, les étudiants peuvent se diriger vers les sites de divers programmes en urologie et le site du CaRMS à partir du site de l'AUC.

Quant aux études supérieures, la catégorie de membre candidat a été créée pour permettre l'inclusion dans la grande famille de l'AUC des résidents et des chercheursboursiers. Cette catégorie de membre inclut un abonnement au JAUC, un rabais substantiel sur les frais d'inscription au congrès annuel, la possibilité de participer à des programmes d'échange avec des associations d'Europe (EUREP) et d'Australie/NouvelleZélande (USANZ), et I'admissibilité à un financement par I'entremise de subventions de recherche et bourses octroyées par la FBAUC. De plus, on a ajouté au JAUC une section intitulée « Resident's Club » invitant les résidents à partager leurs expériences, personnelles et professionnelles, dans le monde professionnel de l'urologie de leur point de vue. Par ailleurs, I'AUC commandite une réunion des résidents seniors en urologie du Canada (RSUC) qui a lieu pendant le congrès annuel de l'AUC. La réunion des RSUC inclut un atelier d'évaluation des manuscrits soumis au JAUC, un cours pratique en urodynamie et la possibilité d'interagir avec des pairs et de réseauter sur le plan professionnel. L'AUC compte aussi un Comité des affaires des résidents qui travaille en collaboration directe avec le Collège royal des médecins et chirurgiens du Canada pour l'établissement de normes de formation en urologie à l'échelle nationale et pour la surveillance du processus lié aux examens de spécialité. Il s'agit là d'un pivot essentiel permettant $d$ 'harmoniser la formation des futurs urologues avec les changements qui se produisent dans l'exercice de la profession.

En plus des initiatives susmentionnées, l'AUC est au courant des inquiétudes concernant le marché du travail au Canada. Le Dr Sid Radomski, en sa qualité de président du Comité socio-économique de l'AUC (maintenant appelé Comité des politiques de santé), a fait un excellent travail préparatoire au cours duquel il a tenté de colliger les meilleures données sur les perspectives d'emploi pour l'avenir à la grandeur du pays. L'AUC a également créé un partenariat avec le Collège royal des médecins et chirurgiens du Canada et d'autres organismes pour examiner la situation de la maind'œuvre en urologie au Canada et formuler des prévisions pour un avenir rapproché.

L'AUC s'est engagée envers les futurs urologues et souhaite se positionner en tant qu'élément essentiel de leur identité. J'encourage tous les urologues en formation et les urologues pratiquants des quatre coins du pays à participer au congrès annuel de I'AUC qui aura lieu à Vancouver, du 25 au 28 juin 2016. Le programme de DPC et le «menu » des activités sociales devraient plaire à tous et garantir l'excellence du congrès.

Je m'en voudrais de ne pas saluer le départ de Josephine Sciortino, directrice de la rédaction du JAUC depuis sa création. $M^{\text {me }}$ Sciortino a été une employée dévouée et loyale de I'AUC et j'aimerais la remercier sincèrement et lui souhaiter beaucoup de succès dans tous ses projets.

$\grave{A}$ tous les membres de l'AUC et leurs familles, j'aimerais souhaiter mes meilleurs vœux pour le temps des Fêtes qui approche à grands pas et une bonne et heureuse année 2016! 